\title{
Amount of Physical Activity in Pregnancy and Infant Heart Outcomes
}

Linda E May ${ }^{1^{*}}$ and Richard R Suminski ${ }^{2}$

${ }^{1}$ Department of Foundational Sciences and Research, East Carolina University (ECU), Greenville, NC 27834, USA

${ }^{2}$ Department of Physiology, KCUMB, Kansas City, MO 64106, USA

*Corresponding author: Linda E. May, Department of Foundational Sciences and Research, East Carolina University (ECU), Greenville, NC 27834, USA, Tel: 252-737-7037; Fax: 252-737-7049; E-mail: mayl@ecu.edu

Rec date: 12 Aug, 2014; Acc date: 10 Oct, 2014; Pub date: 12 Oct, 2014

Copyright: (C) 2014 May L E et al. This is an open-access article distributed under the terms of the Creative Commons Attribution License, which permits unrestricted use, distribution, and reproduction in any medium, provided the original author and source are credited.

\section{Abstract}

Purpose: Our previous findings demonstrated that the amount (dose) of maternal physical activity during pregnancy was associated with changes in fetal heart autonomic control, similar to an adult exercise trained response. The purpose of this study was to determine if the dose of maternal physical activity during association persists after birth.

Methods: We analyzed 43 maternal-infant pairs. Since the majority of measures were not normally distributed, the correlations between infant heart rate (HR) and HR Variability (HRV) metrics and maternal physical activity measures were assessed by the Spearman correlation coefficient. The Spearman procedure also was used to examine relationships among the physical activity variables.

Results: The median values for maternal physical activity duration were $1140 \mathrm{~min}$ ( 90 min/wk), for energy expenditure $6,635 \mathrm{kcal}$, and the intensity was $5.4 \mathrm{kcal} \cdot \mathrm{min}^{-1}$ (Table 1). Based on the average maternal weight, average intensity is near the cut-off $\left(6.0 \mathrm{kcal} . \mathrm{min}^{-1}\right)$ for moderate and vigorous intensity level of physical activity. There was a significant positive correlation between the duration of maternal physical and infant Low Frequency (LF) HRV power $(p=0.02)$, as well as the amount of energy expenditure (i.e. calories burned) in physical activity with infant short-term HRV (RMSSD, $p=0.03)$, LF power $(p=0.01)$ and high frequency $(H F)$ HRV power $(p=0.03)$.

Conclusion: The amount of time and energy expended during physical activity during gestation positively influences the development of neonatal cardiac autonomic control. Coupled with our previous findings of positive fetal dose associated with physical activity during pregnancy suggests, the prenatal period may be the earliest time at which the cardiac health of infants can be positively influenced to decrease risk of heart disease.

Keywords: Neonate; Exercise; Heart rate variability; Heart rate; Pregnancy; Time; Energy expenditure

\section{Introduction}

Clinicians continue to recommend physical activity to adults, and children, for overall heart health benefits, but especially for treating heart disease and attenuating risk factors of heart disease [1]. Physical activity is associated with cardiac changes and decreased incidence of cardiovascular disease, even in pregnant women [2]. While examining the relationship between physical activity and physiological health benefits, it is important to consider the dose response of activity, which includes duration, intensity, and energy expended while active. The dose response of activity is significantly related to increased cardio-respiratory fitness, decreased cardiovascular disease risk, as well as lower resting heart rate (HR) and increased measures of HR variability [3,4]. Lower $\mathrm{HR}$ and increased Heart Rate Variability (HRV), beat-to-beat variation between $\mathrm{R}$ intervals, reflects the beneficial cardiac changes of the Autonomic Nervous System (ANS) balance [5].

In addition to pregnant women gaining cardiovascular benefits from being active during pregnancy [6,7], our previous studies demonstrated that fetal exposure to physical activity during pregnancy was associated with lower fetal HR and increased HRV during maternal rest [8-10]. Furthermore, the duration or amount of maternal physical activity during pregnancy was positively correlated with fetal HRV, while the intensity of physical activity during pregnancy was inversely correlated to fetal HR [11]. Since these changes mirror the physiological adaptations observed in children and adults due to physical activity and are associated with health benefits, it is important to determine if the fetal cardiac changes in response to maternal physical activity during pregnancy persist after birth.

There is growing support of the idea of prenatal programming, which states that exposures during the fetal period influence the health and function in the postnatal period including adulthood [12-17]. Additionally, fetal HR and HRV reflect central and peripheral nervous system integration and are stable measures between the prenatal and postnatal environment [18]. Furthermore, fetal HRV is associated with postnatal temperament [19] and developmental outcomes in early childhood [18] and thus is of clinical importance in pediatric medicine [20].

In light of the idea of prenatal programming and previous findings associating fetal HR/HRV with dose of maternal physical activity during pregnancy, the focus of this investigation was to determine if the association between maternal physical activity during pregnancy and offspring heart outcomes persists into the postnatal period. Our hypothesis was that there is a significant association between dose of 
physical activity (i.e. duration, intensity, energy expenditure) during pregnancy and increased HRV measures of neonates (one-month offspring).

\section{Methods}

The protocol was approved by the Institutional Review Boards (Kansas City University of Medicine and Biosciences and the University of Kansas Medical Center). All subjects provided informed consent prior to participation in the study.

\section{Study population}

We enrolled 66 women in a prospective, non-randomized, nonblinded longitudinal pilot study designed to determine the effects of maternal physical activity during pregnancy on fetal and infant HR and HRV [9]. All participants had healthy singleton pregnancies, were 22-39 years of age, non-smokers with no history of alcohol or drug use. All participants' babies were born healthy. Only forty-three subjects returned with their one-month old infants for a postnatal visit designed as a follow-up to the longitudinal study done during pregnancy [9]. From initial enrollment, attrition mainly due to participant withdrawal, lost contact/moved away, and missed appointment $(\mathrm{n}=20)$; an additional 3 infant recordings were incomplete due to the infant being fussy [21]. All infants were breastfeeding.

\section{Maternal physical activity questionnaire}

The Modifiable Physical Activity Questionnaire (MPAQ) is a reliable and validated questionnaire to assess measures of physical activity during pregnancy [22-24]. Each participant completed the questionnaire at enrollment (about 20 weeks pregnant) and at the end of pregnancy regarding demographics and her duration (minutes) and intensity ( $\mathrm{kcal} /$ minute) of physical activity performed during third trimester of pregnancy. Demographic information included age, prepregnancy weight, height, and calculated pre-pregnancy BMI. Occupational activities or activities of daily living (e.g., getting dressed) were not included since these activities do not elicit a maternal cardiovascular response. Based on previous significant findings related to fetal and infant heart outcomes, only third trimester maternal activity was utilized for calculations [9-11,21]. For dose response calculations, each self-reported aerobic (e.g., walking, jogging), and non-aerobic (e.g., weight lifting, yoga) activity was multiplied by the number of sessions it was performed, then these activity totals were summed for each participant; this provided each participant's 3rd trimester total physical activity duration (minutes). Energy expended (calories burned) was computed (Metabolic Equivalents $\mathrm{X}$ weight $\mathrm{X}$ time) for each physical activity and summed yielding energy expenditure ( $\mathrm{kcal}$ ) for each participant in the $3 \mathrm{rd}$ trimester. Total energy expenditure $(\mathrm{kcal})$ for each participant was then divided by total duration (minutes) providing the average intensity (kcal.min-1) measure [11]. Since pregnancy involves a change in weight, the energy expenditure and intensity values were divided by each participant's body weight (measured with a calibrated scale at 36 weeks gestation) to calculate the relative calories used in maternal physical activities (i.e. weight adjusted energy expenditure).

\section{Infant Magnetocardiogram (MCG) recording}

Magnetocardiogram (MCG) recordings and data processing have been reported previously $[8,25-27]$ for fetus and infant MCG recordings [21]. Women sat next to or held their infant during the 18minute recording with the 83-channel biomagnetometer (CTF Systems Inc., subsidiary of VSM Med Tech, Ltd.). Infants were quiet but alert during recordings. If the infant began to cry, the recording was stopped; a new recording began when the infant was calmed.

\section{Processing infant MCG signals}

Infant MCGs were filtered, and raw signals separated into distinct electrophysiological sources [8, 25-27] similar to previous studies [21] on infants. Using a template-matching algorithm, infant R-peaks were detected to generate the interbeat-interval time-series used for Heart Rate (HR) and Heart Rate Variability (HRV) analyses.

\section{Infant $H R$ and $H R V$ measures}

We used the same metrics as previously reported [9, 21]: HR (bpm); SDNN (the standard deviation of normal R-to-normal $\mathrm{R}$ intervals within the series; overall HRV); RMSSD (the root mean square of successive differences between consecutive interbeat interval series; short-term HRV); LF (low frequency, 0.04-0.2 Hz, power); and HF (high frequency, $0.2-1.7 \mathrm{~Hz}$, power). The ratio of LF and HF (LF/HF) was used as an indicator of sympatho-vagal balance.

\section{Statistical analyses}

Significance level was set a priori at $a<0.05$ and all analyses were done with SAS version 9.2. Since the majority of measures were not normally distributed, the correlations between infant HR and HRV metrics and maternal physical activity measures were assessed by the Spearman correlation coefficient. The Spearman procedure also was used to examine relationships among the physical activity variables.

\section{Results}

\section{Descriptive results}

Of the 43 maternal-infant pairs, three MCG recordings were aborted due to excessive infant fussiness (2 control, 1 exercise group). Complete infant measures and maternal activity data were obtained from 43 maternal-infant pairs. The average participant was 29.0 years \pm 3.9 (mean $\pm \mathrm{SD})$, with a resting heart rate of $89.8 \mathrm{bpm} \pm 11.41$ (means $\pm \mathrm{SD}$ ), had a pre-pregnancy BMI of $24.7 \pm 4.8$ (mean \pm SD), and gained about 14.1 pounds \pm 4.36 (mean \pm SD) during the pregnancy. The median values for maternal physical activity duration were $1140 \mathrm{~min}$ ( $90 \mathrm{~min} / \mathrm{wk})$, for energy expenditure were 6,635 kcal, and the intensity in the $3 \mathrm{rd}$ trimester was $5.4 \mathrm{kcal} . \mathrm{min}-1$. Based on the average maternal weight, average intensity is near the cut-off (6.0 kcal.min ${ }^{-1}$ ) for moderate and vigorous intensity level of physical activity.

All neonates were healthy, full-term infants born $\geq 36$ wks gestation. The average birth weight, length, and ponderal index were all within the normal range for birth values (data not shown). All infant heart measures were within normal range. There were similar numbers of males to females: 20 males and 23 females.

\section{Spearman correlations}

There was a high correlation between weight adjusted and absolute maternal physical activity measures (Table 1). Since factoring body weight into physical activity estimates did not alter the strength of the 
Page 3 of 6

associations with the infant measures, only the results for the absolute physical activity duration, intensity and energy expenditure variables are presented and discussed in the results. All maternal physical activity measures had a negative, but not significant association with infant HR (Table 2); however, these were not significant. Whereas, all maternal physical activity measures had positive associations with all infant HRV measures (Table 2). There were no significant associations between any measure of maternal maternal physical activity and infant sympatho-vagal balance (LF/HF).

\begin{tabular}{|l|l|l|l|l|l|}
\hline & $\begin{array}{l}\text { Duration } \\
(\mathbf{m i n})\end{array}$ & $\begin{array}{l}\text { Energy expenditure } \\
(\mathbf{k c a l})\end{array}$ & $\begin{array}{l}\text { Weight adjusted energy } \\
\text { expenditure (kcal.kg) }\end{array}$ & Intensity (kcal.min-1) & $\begin{array}{l}\text { Weight adjusted intensity } \\
(\mathbf{k c a l} . \mathbf{k g} \cdot \mathbf{m i n}-1)\end{array}$ \\
\hline Duration (min) & & $\begin{array}{l}0.95 \\
(\mathrm{p}<0.0001)\end{array}$ & $0.95(p<0.0001)$ & $0.56(p<0.0001)$ & $0.58(p<0.0001)$ \\
\hline Energy expenditure (kcal) & & & $0.99(\mathrm{p}<0.0001)$ & $0.69(\mathrm{p}<0.0001)$ & $0.69(\mathrm{p}<0.0001)$ \\
\hline Intensity (kcal.min-1) & & & & $0.68(\mathrm{p}<0.0001)$ & $0.70(p<0.0001)$ \\
\hline $\begin{array}{l}\text { Weight adjusted energy } \\
\text { expenditure (kcal.kg.min-1) }\end{array}$ & & & & & $0.91(p<0.0001)$ \\
\hline
\end{tabular}

Table 1: Correlations between Maternal Physical Activity Measures (Min = minutes; $\mathrm{kcal}=$ kilocalories)

\begin{tabular}{|l|l|l|l|}
\hline Infant HR and HRV metrics & Duration $(\mathbf{m i n})$ & Energy expenditure (kcal) & Intensity (kcal.min-1) \\
\hline HR & $\begin{array}{l}r=-0.18(p=0.25) \\
{[-0.45,0.13]}\end{array}$ & $\begin{array}{l}r=-0.15(p=0.33) \\
{[-0.43,0.16]}\end{array}$ & $\begin{array}{l}r=-0.06(p=0.71) \\
{[-0.35,0.25]}\end{array}$ \\
\hline SDNN & $\begin{array}{l}r=0.23(p=0.15) \\
{[-0.08,0.49]}\end{array}$ & $\begin{array}{l}r=0.26(p=0.09) \\
{[-0.04,0.52]}\end{array}$ & $\begin{array}{l}r=0.17(p=0.26) \\
{[-0.13,0.45]}\end{array}$ \\
\hline RMSSD & $\begin{aligned} r=0.29(p=0.06) \\
{[-0.01,0.54] }\end{aligned}$ & $\begin{array}{l}r=0.33(p=0.03) \\
{[0.03,0.57]}\end{array}$ & $\begin{array}{l}r=0.15(p=0.35) \\
{[-0.16,0.43]}\end{array}$ \\
\hline LF & $r=0.35(p=0.02)$ & $\begin{array}{l}r=0.38(p=0.01) \\
{[0.08,0.61]}\end{array}$ & $\begin{array}{l}r=0.24(p=0.12) \\
{[-0.07,0.50]}\end{array}$ \\
\hline HF & {$[0.05,0.59]$} & $\begin{array}{l}r=0.34(p=0.03) \\
{[0.04,0.58]}\end{array}$ & $\begin{array}{l}r=0.16(p=0.29) \\
{[-0.15,0.44]}\end{array}$ \\
\hline LF/HF & $r=0.28(p=0.07)$ & $r=-0.03(p=0.85)$ & $r=-0.12(p=0.46)$ \\
\hline
\end{tabular}

Table 2: Correlations between Infant HR and HRV Metrics and Maternal Physical Activity Measures [95\% confidence intervals] (HR - heart rate; HRV - heart rate variability; SDNN - standard deviation of normal-to-normal intervals (overall HRV); RMSSD -root mean square of successive differences (short-term HRV); LF - low frequency; HF - high frequency; LF/HF - an index of sympatho-vagal balance)

\section{Duration of maternal physical activity}

There was a significant positive correlation between the duration of maternal physical and infant LF power $(\mathrm{p}=0.02)$, a measure of parasympathetic and sympathetic control (Table 2). Infant short-term HRV (RMSSD, $\mathrm{p}=0.06)$ and HF power $(\mathrm{p}=0.07)$ were also associated with maternal duration of physical activity; however, these results just missed the criteria for significance.

\section{Energy expended from maternal physical activity}

The amount of energy expenditure (i.e. calories burned) in physical activity was positively correlated with infant short-term HRV (RMSSD, $\mathrm{p}=0.03$ ), LF power $(\mathrm{p}=0.01)$ and HF power $(\mathrm{p}=0.03)$ (Table 2 ). The association between maternal energy expenditure during physical activity and infant overall HRV approached significance (SDNN, $\mathrm{p}=0.09$ ).

\section{Intensity of maternal physical activity}

Unlike our findings during the fetal period, there were no significant associations between maternal intensity of physical activity and infant HR (Table 2). No significant correlations were found between maternal physical intensity and infant HRV measures. None of these findings survived the threshold corrected by Bonferroni or Benjamini-Hochburg's methods for multiple comparisons.

\section{Discussion}

These data support the general hypothesis of the persistence of the dose response relationship maternal physical activity with the infant cardiac measures [11]. Specifically, the duration of maternal physical activity was positively correlated with infant LF power, parasympathetic and sympathetic measure. Maternal energy expenditure was positively associated with indicators of both branches of the autonomic nervous system (LF and HR power, RMSSD). These 
findings are similar, but different to the previous findings in fetal measures of autonomic control.

The significant changes in cardiac autonomic control in response to the amount (dose) of time spent in physical activity are similar to those reported in the fetus [11]. Fetal findings demonstrated amount (dose) of time women spent in physical activity was associated with changes in fetal cardiac parasympathetic and sympathetic measures of very low frequency power of HRV (VLF), LF, HF, RMSSD, and SDNN [11]. Most likely the difference in significant findings between the fetal and infant data is related to two things: a smaller sample size and sympathetic dominance. During the neonatal period, the heart undergoes numerous changes as it transitions from the fetal cardiovascular circulation to the adult-type circulation [28]. During this specific time of adaptation to the external environment the sympathetic nervous system influence on the heart is higher than at any point of development [28]. This developmental shift along with a smaller sample size can explain why significant differences are seen in a measure of sympathetic tone, and was just below significance in measures of parasympathetic control (RMSSD, HF). Nonetheless, the important message is for pregnant women to do at least some physical activity.

Significant associations seen in infant cardiac autonomic control in response to the amount (dose) of energy expended in physical activity was not previously reported in in the fetus [11]. However, fetal findings demonstrated the intensity of women while being physically activity was associated with significant changes in fetal cardiac sympathetic measure of VLF [11] while infant measures found differences in LF (parasympathetic and sympathetic measure), and RMSSD with HR (both measures of parasympathetics) [21]. Although these are different measures, energy expended takes in to account the amount of time doing an activity (i.e. 30 minutes vs. 60 minutes) as well as how difficult the activity is (i.e. walking vs. running); thus it seems time and intensity of maternal activity are important factors in fetal and neonatal cardiac autonomic development. Interestingly, the current findings are similar to those seen in a diverse group of young adults after a 12-week aerobic training program [29]. Our current findings fully support current guidelines from the American Congress of Obstetrics and Gynecology, the Royal College of Obstetricians and Gynaecologists, and the Society of Obstetricians and Gynecologists of Canada, which now suggest sedentary pregnant women start an exercise program, at least 15 minutes of moderate intensity aerobic exercise 3 times a week, then progressing to 30 -minute sessions 4 to 7 times a week [30-32].

The preservation of a specific developmental trajectory from prenatal to postnatal life supports the developmental origins hypothesis [6,12,33-35] and suggests that physical activity during pregnancy has a positive prenatal programming effect on offspring heart development [18]. Growing evidence demonstrates that people who develop cardiovascular disease had a different in utero environment so that birthweight tended to be at the lower end of normal range and this persisted through infancy into early childhood as well $[15,33,36-40]$. Considering the developmental model of prenatal programming and current findings along with previous findings relating exercise training to decreased incidence of cardiovascular disease [41], the implications support prenatal activity as the earliest intervention for treating/preventing heart disease. Previously, heart rate variability has been used as an essential tool for studying the autonomic disturbance in adult and pediatric patients with cardiac diseases [42-44]. However, this study supports the use of heart rate variability as a sensitive clinical marker for cardiovascular health and disease in the neonatal population. Many of the HRV indices are simple to compute, or can be done with inexpensive software upgrades, and do not require any special procedures for Holter monitoring or standard ECG recordings [42], though the benefit to clinician and the patient are invaluable.

There are limitations to our study. Although the questionnaire used is reliable and validated for assessing physical activity in pregnant women $[23,24,45,46]$; it is a self-report instrument that is vulnerable to recall bias and could lead to inflated estimations of physical activity levels [47]. Secondly, women who are physically active during pregnancy may have had improved health fitness measures prior to becoming pregnant and that level of fitness may influence our findings. Current research is underway to address the potential influence of maternal pre-pregnancy fitness on fetal and infant heart outcomes. Further, we did not control for other potential confounding variables known to influence fetal development such as maternal diet. Future studies will need to control for maternal nutrient intake throughout pregnancy.

In conclusion, we previously demonstrated that maternal physical activity had a dose response relationship with fetal cardiac autonomic measures and current analyses demonstrate these differences persist into the postnatal period, up to one-month of age. Our new findings suggest that the amount of time women are physical activity during a healthy, low-risk pregnancy can have a potentially positive influence on cardiac autonomic development of the offspring after birth. Additionally, the amount of effort expended during the activity has a dose response as well, suggesting women should exercise at moderate intensity and may have offspring with increased autonomic development compared to those who exercised at light intensity. In light of the developmental model of prenatal programming and the current findings, prenatal physical activity may be the earliest intervention for preventing heart disease in neonates. Furthermore, the continued use of HRV measures are clinically relevant for determine disease states, such as heart disease or obesity; as well as heart health and indices of a training response. These findings have great public health impact for the cardiovascular health and decrease risk of heart disease in future generations.

\section{Acknowledgements}

The authors are grateful to the women who participated in this study. We would also like to Dr. Gustafson and her staff (JoAnn Liermann, RNC, PhD and Lori Blanck, R. EEG/EP T) for their assistance with data acquisition and processing. The authors recognize MihaiPopescu, PhD and E. AndaPopescu, PhD for their contribution and expertise in designing the MatLab routines for FHR and HRV analyses. The results of the present study do not constitute endorsement by ACSM.

\section{Financial Support}

This study was supported in part by pilot funding from the Hoglund Brain Imaging Center, through a generous gift from Forrest and Sally Hoglund, an intramural grant from the Kansas City University of Medicine and Biosciences (May), and the Kansas Intellectual Development and Disabilities Research Center (P30 NICHD HD 002528). K.M. Gustafson is supported in part by NICHD R21 HD059019 and R01 HD047315. 


\section{References}

1. Kones R (2011) Primary prevention of coronary heart disease: integration of new data, evolving views, revised goals, and role of rosuvastatin in management. A comprehensive survey. Drug Des DevelTher 5: 325-380.

2. Manson JE, Hu FB, Rich-Edwards JW, Colditz GA, Stampfer MJ, et al. (1999) A prospective study of walking as compared with vigorous exercise in the prevention of coronary heart disease in women. N Engl J Med 341: 650-658.

3. Hamer M, Chida Y (2008) Active commuting and cardiovascular risk: a meta-analytic review. Prev Med 46: 9-13.

4. Shephard RJ, Balady GJ (1999) Exercise as cardiovascular therapy Circulation 99: 963-972

5. Akselrod S, Gordon D, Madwed JB, Snidman NC, Shannon DC, et al. (1985) Hemodynamic regulation: investigation by spectral analysis. Am J Physiol 249: H867-875.

6. Clapp JF (2000) Exercise during pregnancy. A clinical update. Clin Sports Med 19: 273-286.

7. Clapp JF, Capeless E (1991) The VO2max of recreational athletes before and after pregnancy. Med Sci Sports Exerc 23: 1128-1133.

8. Gustafson KM, May LE, Yeh HW, Million SK, Allen JJ (2012) Fetal cardiac autonomic control during breathing and non-breathing epochs: the effect of maternal exercise. Early Hum Dev 88: 539-546.

9. May LE, Glaros A, Yeh HW, Clapp JF 3rd, Gustafson KM (2010) Aerobic exercise during pregnancy influences fetal cardiac autonomic control of heart rate and heart rate variability. Early Hum Dev 86: 213-217.

10. May LE, Suminski RR, Berry A, Langaker MD, Gustafson KM (2014) Maternal physical activity mode and fetal heart outcome. Early Hum Dev 90: 365-369.

11. May LE, Suminski RR, Langaker MD, Yeh HW, Gustafson KM (2012) Regular maternal exercise dose and fetal heart outcome. Med Sci Sports Exerc 44: 1252-1258.

12. Barker DJ, Fall CH (1993) Fetal and infant origins of cardiovascular disease. Arch Dis Child 68: 797-799.

13. Barker DJ, Osmond C (1986) Infant mortality, childhood nutrition, and ischaemic heart disease in England and Wales. Lancet 1: 1077-1081.

14. Barker DJ, Osmond C, Golding J, Kuh D, Wadsworth ME (1989) Growth in utero, blood pressure in childhood and adult life, and mortality from cardiovascular disease. BMJ 298: 564-567.

15. Barker DJ, Osmond C, Law CM (1989) The intrauterine and early postnatal origins of cardiovascular disease and chronic bronchitis. J Epidemiol Community Health 43: 237-240.

16. Hales C N, BarkerDJ (1992) Type 2 (non-insulin-dependent) diabetes mellitus: the thrifty phenotype hypothesis. Diabetologia 35: 595-601.

17. Barker DJ, Bagby SP, Hanson MA (2006) Mechanisms of disease: in utero programming in the pathogenesis of hypertension. Nat ClinPractNephrol 2: $700-707$.

18. DiPietro JA, Bornstein MH, Hahn CS, Costigan K, Achy-Brou A (2007) Fetal heart rate and variability: stability and prediction to developmental outcomes in early childhood. Child Dev 78: 1788-1798.

19. DiPietro JA, Ghera MM, Costigan KA (2008) Prenatal origins of temperamental reactivity in early infancy. Early Hum Dev 84: 569-575.

20. Porges SW, Furman SA (2011) The Early Development of the Autonomic Nervous System Provides a Neural Platform for Social Behavior: A Polyvagal Perspective. Infant Child Dev 20: 106-118.

21. May LE, Scholtz SA, Suminski R, Gustafson KM (2014) Aerobic exercise during pregnancy influences infant heart rate variability at one month of age. Early Hum Dev 90: 33-38.

22. Cramp AG, BrawleyLR (2009) Sustaining self-regulatory efficacy and psychological outcome expectations for postnatal exercise: effects of a group-mediated cognitive behavioural intervention. Br J Health Psychol 14: 595-611

23. Cramp AG, Bray SR (2009) A prospective examination of exercise and barrier self-efficacy to engage in leisure-time physical activity during pregnancy. Ann Behav Med 37: 325-334.
24. Cramp AG, Bray SR (2009) Pre- and postnatal women's leisure time physical activity patterns: a multilevel longitudinal analysis. Res Q Exerc Sport 80: 403-411.

25. Gustafson KM, Allen JJ, Yeh HW, May LE (2011) Characterization of the fetal diaphragmatic magnetomyogram and the effect of breathing movements on cardiac metrics of rate and variability. Early Hum Dev 87: 467-475

26. Popescu EA, Popescu M, Bennett TL, Lewine JD, Drake WB, et al. (2007) Magnetographic assessment of fetal hiccups and their effect on fetal heart rhythm. PhysiolMeas 28: 665-676.

27. Popescu EA, Popescu M, Wang J, Barlow SM, Gustafson KM (2008) Non-nutritive sucking recorded in utero via fetal magnetography. PhysiolMeas 29: 127-139.

28. Blackburn S (2013) Maternal, Fetal, and Neonatal Physiology. 4th ed, A clinical perspective, Saunders, Maryland Heights 768.

29. Grant CC, Viljoen M, Janse van Rensburg DC, Wood PS (2012) Heart rate variability assessment of the effect of physical training on autonomic cardiac control. Ann Noninvasive Electrocardiol 17: 219-229.

30. ACOG Committee Obstetric Practice (2002) ACOG Committee opinion Number 267, January 2002: exercise during pregnancy and the postpartum period. ObstetGynecol 99: 171-173.

31. Bell B, Dooley MM (2012) Exercise In Pregnancy. Royal College of Obstetricians and Gynaecologists 4: 7.

32. Davies GA (2003) Society of Obstetricians and Gynecologists of Canada, SOGC Clinical Practice Obstetrics Committee. Joint SOGC/CSEP Clinical Practice Guideline: Exercise in Pregnancy and the Postpartum Period. Canadian Journal of Applied Physiology 28: 11.

33. Barker DJ (1995) Fetal origins of coronary heart disease. BMJ 311: 171-174.

34. Barker DJ (1997) Intrauterine programming of coronary heart disease and stroke. ActaPaediatrSuppl 423: 178-182.

35. Barker DJ (1997) Intra-uterine programming of the adult cardiovascular system. CurrOpinNephrolHypertens 6: 106-110.

36. Osmond C, Barker DJ, Winter PD, Fall CH, Simmonds SJ (1993) Early growth and death from cardiovascular disease in women. BMJ 307: 1519-1524.

37. Rich-Edwards JW, Stampfer MJ, Manson JE, Rosner B, Hankinson SE, et al. (1997) Birth weight and risk of cardiovascular disease in a cohort of women followed up since 1976. BMJ 315: 396-400.

38. Stein CE, Fall CH, Kumaran K, Osmond C, Cox V, et al. (1996) Fetal growth and coronary heart disease in south India. Lancet 348: 1269-1273.

39. Stein PK, Ehsani AA, Domitrovich PP, Kleiger RE, Rottman JN (1999) Effect of exercise training on heart rate variability in healthy older adults. Am Heart J 138: 567-576.

40. Leon DA, Lithell HO, Vâgerö D, Koupilová I, Mohsen R, et al. (1998) Reduced fetal growth rate and increased risk of death from ischaemic heart disease: cohort study of 15000 Swedish men and women born 1915-29. BMJ 317: 241-245.

41. Manson JE, Greenland P, LaCroix AZ, Stefanick ML, Mouton CP, et al. (2002) Walking compared with vigorous exercise for the prevention of cardiovascular events in women. N Engl J Med 347: 716-725.

42. Massin MM (1999) Heart rate variability in healthy and cardiac children. Am J Cardiol 84: 1278.

43. Massin MM, Derkenne B, Tallsund M, Rocour-Brumioul D, Ernould C, et al. (1999) Cardiac autonomic dysfunction in diabetic children. Diabetes Care 22: 1845-1850.

44. Massin MM, Derkenne B, von Bernuth G (1999) Correlations between indices of heart rate variability in healthy children and children with congenital heart disease. Cardiology 91: 109-113.

45. Kriska AM, Bennett PH (1992) An epidemiological perspective of the relationship between physical activity and NIDDM: from activity assessment to intervention. Diabetes Metab Rev 8: 355-372.

46. Kriska AM, Knowler WC, LaPorte RE, Drash AL, Wing RR, et al. (1990) Development of questionnaire to examine relationship of physical activity and diabetes in Pima Indians. Diabetes Care 13: 401-411. 
Citation: May L E, Suminski R R (2014) Amount of Physical Activity in Pregnancy and Infant Heart Outcomes. J Neonatal Biol 3: 160. doi: 10.4172/2167-0897.1000160

Page 6 of 6

47. Saelens BE, Sallis JF, Black JB, Chen D (2003) Neighborhood-based differences in physical activity: an environment scale evaluation. Am J Public Health 93: 1552-1558. 